\title{
Facile and Large Scale Synthesis of Diverse 4-O-Protected 2,3-O-Isopropylidene-D-erythrose
}

\author{
Sukumar Bepary, ${ }^{\dagger}$ In Kwon Yoon, ${ }^{\dagger}$ and Ge Hyeong Lee* \\ Korea Research Institute of Chemical Technology, P.O. Box 107, Yusung, Taejeon 305-600, Korea \\ *E-mail: ghlee@krict.re.kr \\ ${ }^{\dagger}$ Pai Chai University, Department of Chemistry, Seo-Gu Domadong 439-6, Taejeon 302-160, Korea \\ Received July 1, 2010, Accepted September 24, 2010
}

Key Words: Chiral, Enantiomer, Erythrose, Building block

Enantiomerically pure building blocks with two chiral centers play important roles in the total synthesis of naural compounds ${ }^{1}$ as well as the synthesis of biologically important compounds. ${ }^{2}$ One of these type of chiral building blocks is $2,3-O$ isopropylidene-D-erythrose, whose derivatives are already of great interests to the researchers to achieve the synthetic targets. ${ }^{3}$ The aldehyde functionalities, similar to that of erythrose moiety, have attracted the synthetic chemists as it permits convenient addition of a lot of other functional groups thereby allowing diversified organic molecules. ${ }^{4}$ On the other hand, the hydroxy functional groups, similar to 4-hydroxy group of erythrose, are also interesting to connect other groups for getting diversity in the structures. ${ }^{5}$ The additional scope of convenient generation of glycol moiety by the release of the 2,3-O-protective isopropyledene group stimulated considerable interests in biosynthetic and pharmacological studies, as well as in the total synthesis of natural compounds. ${ }^{6}$ Moreover, the scope of simple conversion of the configuration from cis to trans may provoke additional synthetic interests to the researchers as this type of conversion is already in use. ${ }^{7}$ Thus 4- $O$-protected 2,3-O-isopropylideneD-erythrose appears to be an important chiral building block for the synthetic and medicinal chemists.
Though synthesis of 4-O-protected 2,3-O-isopropylideneD-erythrose is possible by opening the lactol ring to get alcohol (6) which can be converted to 4-O-protected alcohol (7). This alcohol can be subjected to ozonolysis to get the desired D-erythrose (8) by following the already published procedures (Scheme 1). But because of significant limitations such as lack of convenience, long reaction time, and low to moderate yield, sometimes those may be of discouraging for the researchers. ${ }^{8}$ In this paper, we report a convenient and efficient method for the synthesis of enantiomerically pure 4-O-protected 2,3-Oisopropylidene-D-erythrose.

\section{Results and Discussion}

By following the procedure published by Li, Y. L. et al., ${ }^{9}$ commercially available D-(-)-isoascorbic acid (5) was converted to (-)-2,3-O-isopropylidene-D-erythronolactol (4), which on subsequent refluxing with $N, N$-dimethylhydrazine in absolute ethanol for 3 hours afforded the hydrazone (3) in 96\% yield. The reaction was observed to be very clean, thereby permitting one-pot reaction with the respective halides in presence of a base to provide diverse 4- $O$-protected derivatives (2a-d) in excel-

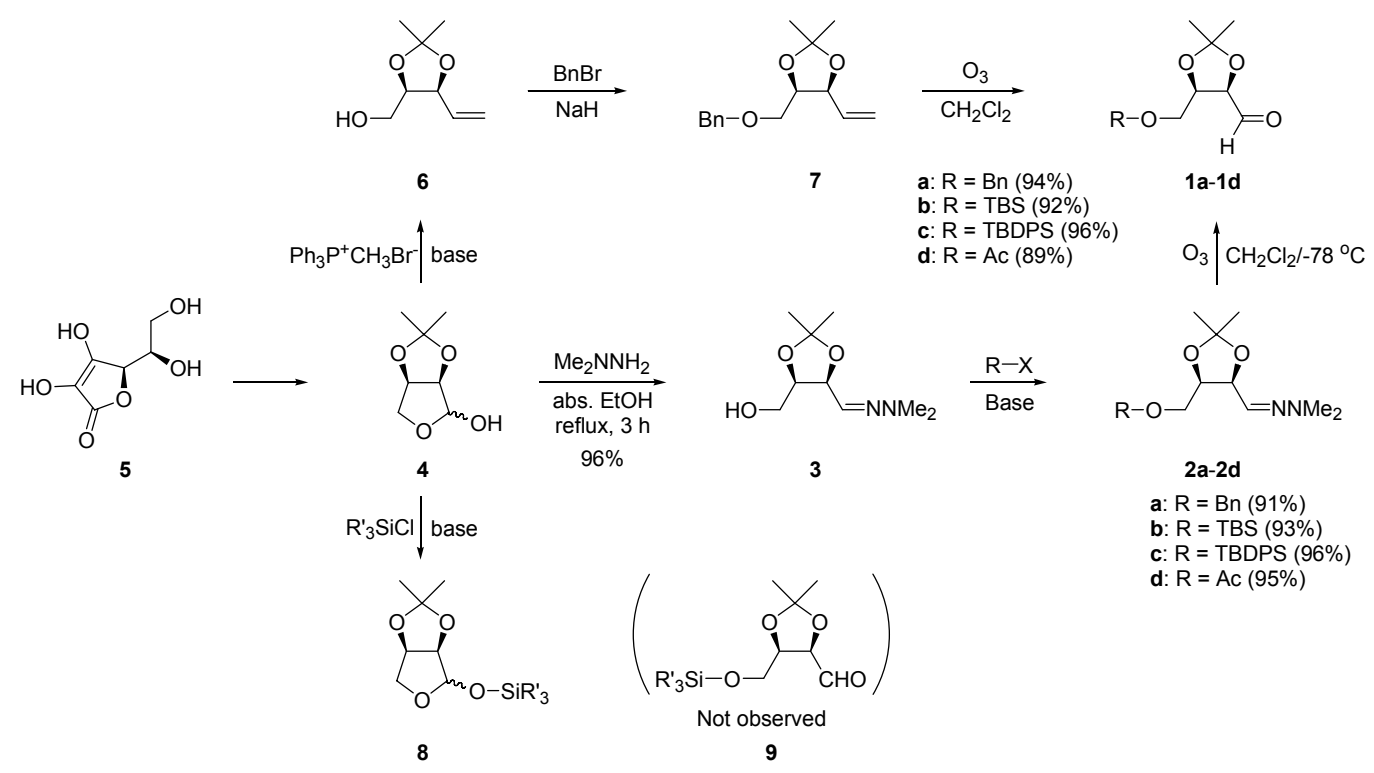

Scheme 1. Synthesis of diverse 4-O-protected 2,3-O-isopropylidene- D-erythrose 
lent yields (91 - 96\%). Subsequent ozonolysis of these 4-O-protected derivatives afforded the desired carboxaldehydes (1a-d) in $89-96 \%$ yields (Scheme 1).

It was reported that the ring opening reaction of erythronolactol (4) offered the alcohol (6), but required more than 2 equiv. of triphenylphosphonium bromide resulting in the product contaminated by triphenylphosphine oxide. ${ }^{8}$ Moreover, the reaction required relatively long time (12 hours), but showed variable yields $(75-99 \%)$. In addition, ozonolysis of the protected alcohol (7) gave poor yield (45\%) of the desired products (1a). ${ }^{10}$ Again, though it was published ${ }^{11}$ that silyl chlorides can open the lactol ring resulting in 4-O-protected aldehydes (9), several trials in our lab with TBSCl, TESCl, and TBDPSCl were not sussessful to offer the open form (9), but to generate the silyl derivataives (8) exclusively. Thus our method conveys the benefits over these published methods since it requires short time (3 hours) but represents efficient and convenient one-pot reaction with excellent overall yields. In addition, the highly volatile by-product, $N$-nitroso- $N, N$-dimethylamine, in the ozonolysis of the 4-O-protected hydrazones was evaporated conveniently on a rotary evaporator. Spectra and optical rotation values of all the products were found to be similar to those of the reported compounds. ${ }^{12-15}$

\section{Experimental Section}

General. ${ }^{1} \mathrm{H}$ NMR spectra were recorded at $300 \mathrm{MHz}$, unless otherwise specified, in $\mathrm{CDCl}_{3}$ solution using tetramethylsilane as internal standard. Analytical thin-layer chromatography was performed on precoated silica gel plates $(0.25-\mathrm{mm} 60 \mathrm{~F}-254$ E. Merck). The products were purified by flash column chromatography on silica gel 60 (Merck, 230 - 400 mesh). THF was dried over sodium benzophenone prior to use. Commercially available compounds and solvents were used without previous purification.

(4R,5S)-5-(Hydroxymethyl)-2,2-dimethyl-1,3-dioxolane4-carbaldehyde $\mathbf{N}, \mathbf{N}$-dimethyl-hydrazone (3). A stirred solution of (-)-2,3-O-isopropylidene-D-erythronolactol $(30.0 \mathrm{mmol})$ and $N, N$-dimethylhydrazine $(36.0 \mathrm{mmol})$ in absolute $\mathrm{EtOH}(50$ $\mathrm{mL}$ ) was refluxed for $3 \mathrm{~h}$. The reaction mixture was allowed to cool to room temperature and concentrated in vacuo to afford crude product as very pale yellow oil, which was used without further purification. Analytical sample was obtained by flash column chromatography using 20 - 40\% ethyl acetate in $n$-hexane as eluent to give pure product as colorless oil.

Yield: $96 \%$, colorless viscous oil. ${ }^{1} \mathrm{H}$ NMR $\left(\mathrm{CDCl}_{3}\right) \delta 1.39$ (s, $3 \mathrm{H}), 1.40$ (s, $3 \mathrm{H}), 2.83(\mathrm{~s}, 6 \mathrm{H}), 3.66(\mathrm{~m}, \mathrm{OH}$ and $2 \mathrm{H}), 4.34$ $(\mathrm{m}, 1 \mathrm{H}), 4.80(\mathrm{~m}, 1 \mathrm{H}), 6.49(\mathrm{~d}, 1 \mathrm{H}, J=6.5 \mathrm{~Hz})$.

(4R,5S)-5-(Benzyloxymethyl)-2,2-dimethyl-1,3-dioxolane4-carbaldehyde $\mathbf{N}, \mathbf{N}$-dimethyl-hydrazone (2a). To a stirred mixture of $\mathrm{NaH}$ ( $60 \%$ dispersion in mineral oil, $16.5 \mathrm{mmol})$ in dry THF $(40 \mathrm{~mL})$ was added dropwise a solution of alcohol $(15.0 \mathrm{mmol})$ in dry THF $(10 \mathrm{~mL})$ over $10 \mathrm{~min}$ at room temperature. After $30 \mathrm{~min}$, benzyl bromide $(16.5 \mathrm{mmol})$ was added. After $3 \mathrm{~h}$ more stirring, the reaction mixture was poured into cold water and extracted with ethyl acetate, washed with brine, dried over $\mathrm{MgSO}_{4}$, filtered, and then concentrated in vacuo to afford crude product, which was purified by flash column chro- matography using $10-20 \%$ ethyl acetate in $n$-hexane as eluent to give pure product as colorless oil.

Yield: 91\%, colorless viscous oil. ${ }^{1} \mathrm{H}$ NMR $\left(\mathrm{CDCl}_{3}\right) \delta 1.38$ (s, $3 \mathrm{H}), 1.51$ (s, $3 \mathrm{H}), 2.77$ (s, $6 \mathrm{H}), 3.49$ (dd, $1 \mathrm{H}, J=7.3,10.5$ $\mathrm{Hz}), 3.55$ (m, 1 H), 4.42 (m, 1 H), 4.57 (ABq, $2 \mathrm{H}), 4.74$ (m, $1 \mathrm{H}), 6.40$ (d, $1 \mathrm{H}, J=7.3), 7.10-7.45(\mathrm{~m}, 5 \mathrm{H})$.

$(4 R, 5 S)-5$-[(tert-Butyldimethylsilyloxy)methyl]-2,2-dimethyl-1,3-dioxolane-4-carbaldehyde $\mathrm{N}, \mathrm{N}$-dimethylhydrazone (2b). A mixed solution of alcohol (15.0 mmol), tert-butylchlorodimethylsilane $(16.5 \mathrm{mmol})$, and imidazole $(20.0 \mathrm{mmol})$ in dry dichloromethane $(60 \mathrm{~mL})$ was stirred for $2 \mathrm{~h}$ at $0{ }^{\circ} \mathrm{C}$. The reaction mixture was concentrated in vacuo and the sticky residue was diluted with ethyl acetate and water. The organic layer was separeted, washed with $0.5 \mathrm{~N} \mathrm{HCl}$ and saturated aqueous $\mathrm{NaHCO}_{3}$ solution, dried over $\mathrm{MgSO}_{4}$, filtered, and then concentrated in vacuo to afford crude product, which was purified by flash column chromatography using $10-20 \%$ ethyl acetate in $n$-hexane as eluent to give pure product as colorless oil.

Yield: 93\%, colorless viscous oil. ${ }^{1} \mathrm{H}$ NMR $\left(\mathrm{CDCl}_{3}\right) \delta 0.00$ $(\mathrm{s}, 6 \mathrm{H}), 0.82(\mathrm{~s}, 9 \mathrm{H}), 1.31(\mathrm{~s}, 3 \mathrm{H}), 1.43(\mathrm{~s}, 3 \mathrm{H}), 2.76(\mathrm{~s}, 6 \mathrm{H})$, $3.57(\mathrm{dd}, 1 \mathrm{H}, J=7.3,10.9 \mathrm{~Hz}), 3.67(\mathrm{~m}, 1 \mathrm{H}), 4.16(\mathrm{~m}, 1 \mathrm{H})$, $4.68(\mathrm{~m}, 1 \mathrm{H}), 6.67$ (d, $1 \mathrm{H}, J=7.3)$.

(4R,5S)-5-[(tert-Butyldiphenylsilyloxy)methyl]-2,2-dimethyl-1,3-dioxolane-4-carbaldehyde $\mathrm{N}, \mathrm{N}$-dimethylhydrazone (2c). Following the above procedure, product was obtained as colorless oil.

Yield: $96 \%$, colorless viscous oil. ${ }^{1} \mathrm{H}$ NMR $\left(\mathrm{CDCl}_{3}\right) \delta 1.04$ (s 9H), 1.37 (s $3 \mathrm{H}), 1.48$ (s, $3 \mathrm{H}), 2.77$ (s, $6 \mathrm{H}), 3.68$ (dd, $1 \mathrm{H}$, $J=7.3,10.8 \mathrm{~Hz}), 3.80(\mathrm{~m}, 1 \mathrm{H}), 4.26(\mathrm{~m}, 1 \mathrm{H}), 4.78(\mathrm{~m}, 1 \mathrm{H})$, $6.52(\mathrm{~d}, 1 \mathrm{H}, J=7.3 \mathrm{~Hz}), 7.41-7.66(\mathrm{~m}, 10 \mathrm{H})$.

(4R,5S)-5-(Acetyloxymethyl)-2,2-dimethyl-1,3-dioxolane4-carbaldehyde $\mathbf{N}, \mathbf{N}$-dimethyl-hydrazone (2d). A mixed solution of alcohol $(15.0 \mathrm{mmol})$, acetic anhydride (16.5 mmol), triethylamine $(50.0 \mathrm{mmol})$, and catalytic amount of DMAP in dry dichloromethane $(60 \mathrm{~mL})$ was stirred for $3 \mathrm{~h}$ at $0{ }^{\circ} \mathrm{C}$. The reaction mixture was concentrated in vacuo to afford crude product, which was purified by flash column chromatography using $10-20 \%$ ethyl acetate in $n$-hexane as eluent to give pure product as colorless oil.

Yield: 95\%, colorless viscous oil. ${ }^{1} \mathrm{H}$ NMR $\left(\mathrm{CDCl}_{3}\right) \delta 1.39$ $(\mathrm{s}, 3 \mathrm{H}), 1.52(\mathrm{~s}, 3 \mathrm{H}), 2.08(\mathrm{~s}, 3 \mathrm{H}), 2.83(\mathrm{~s}, 6 \mathrm{H}), 4.00(\mathrm{dd}, 1 \mathrm{H}$, $J=7.3,11.6 \mathrm{~Hz}), 4.26(\mathrm{~m}, 1 \mathrm{H}), 4.43(\mathrm{~m}, 1 \mathrm{H}), 4.79(\mathrm{~m}, 1 \mathrm{H})$, $6.33(\mathrm{~d}, 1 \mathrm{H}, J=7.3)$.

Ozonolysis for the synthesis of 4- $\mathrm{O}$-protected 2,3-O $\mathrm{O}$-isopropylidene-D-erythrose (1a-1d). A stirred solution of protected hydrazone $(10.0 \mathrm{mmol})$ in anhydrous dichloromethane (50 $\mathrm{mL}$ ) was ozonolysized at $-78^{\circ} \mathrm{C}$. After hydrazone disappeared on TLC, the reaction mixture was quenched with dimethyl sulfide $(5 \mathrm{~mL})$ and allowed to warm to room temperature. After $2 \mathrm{~h}$ more stirring at room temperature, the reaction mixture was concentrated in vacuo to afford viscous oil, which was purified by flash column chromatography using $10-20 \%$ ethyl acetate in $n$-hexane as eluent to give pure products.

4- $\boldsymbol{O}$-Benzyl-2,3- $\boldsymbol{O}$-isopropylidene-D-erythrose (1a). ${ }^{12}$ Yield: $94 \%$, colorless viscous oil; $[\alpha]_{\mathrm{D}}^{25}+19.3^{\circ}\left(c 1.34, \mathrm{CHCl}_{3}\right)\left[\right.$ lit. $^{13}$ L-isomer; $[\alpha]_{\mathrm{D}}^{26}-18^{\mathrm{o}}\left(c\right.$ 1.34, $\left.\left.\mathrm{CHCl}_{3}\right)\right] ;{ }^{1} \mathrm{H} \mathrm{NMR}\left(\mathrm{CDCl}_{3}\right) \delta 1,39$ (s, $3 \mathrm{H}, \mathrm{Me}) .1 .36$ (s, $3 \mathrm{H}), 1.55$ (s, $3 \mathrm{H}), 3.44$ (dd, $J=10.7,3.9$ $\mathrm{Hz}, 1 \mathrm{H}), 3.65$ (dd, $J=10.7,3.8 \mathrm{~Hz}, 1 \mathrm{H}), 4.40-4.65$ (m, $2 \mathrm{H})$, 
7,25-7,30 (m, 5H), 9,63 (d, $J=2.1 \mathrm{~Hz}, 1 \mathrm{H})$.

4-O-tert-Butyldimethylsilyl-2,3-O-isopropylidene-D-eryt hrose (1b). ${ }^{13}$ Yield: $92 \%$, colorless viscous oil; $[\alpha]_{\mathrm{D}}^{25}+52.3^{\circ}(c$ $\left.1.2, \mathrm{CHCl}_{3}\right)\left[\right.$ lit. $\left.^{14}[\alpha]_{\mathrm{D}}^{20}+51.8^{\mathrm{o}}\left(c 1.2, \mathrm{CHCl}_{3}\right)\right] ;{ }^{1} \mathrm{HNMR}\left(\mathrm{CDCl}_{3}\right)$ $\delta 0.00(\mathrm{~s}, 3 \mathrm{H}), 0.04(\mathrm{~s}, 3 \mathrm{H}), 0.84(\mathrm{~s}, 9 \mathrm{H}), 1.34(\mathrm{~s}, 3 \mathrm{H}), 1.52$ $(\mathrm{s}, 3 \mathrm{H}), 3.68(\mathrm{~m}, 1 \mathrm{H}), 3.73(\mathrm{dd}, 1 \mathrm{H}, J=2.1,11.3 \mathrm{~Hz}), 4.41(\mathrm{~m}$, $2 \mathrm{H}), 9.63(\mathrm{~d}, 1 \mathrm{H}, J=2.1 \mathrm{~Hz})$.

4-O-tert-Butyldiphenylsilyl-2,3-O-isopropylidene-D-eryth rose (1c). ${ }^{14}$ Yield: $96 \%$, colorless viscous oil; $[\alpha]_{\mathrm{D}}^{25}+41.9^{\circ}(c 1.0$, $\left.\mathrm{CHCl}_{3}\right)\left[\right.$ lit. $\left.^{15}[\alpha]_{\mathrm{D}}^{25}+43.3^{\circ}\left(c 1.01, \mathrm{CHCl}_{3}\right)\right] ;{ }^{1} \mathrm{H} \mathrm{NMR}\left(\mathrm{CDCl}_{3}\right)$ $\delta 1.02(\mathrm{~s}, 9 \mathrm{H}), 1.39(\mathrm{~s}, 3 \mathrm{H}), 1.61(\mathrm{~s}, 3 \mathrm{H}), 3.72(\mathrm{~m}, 1 \mathrm{H}), 3.82$ (dd, $1 \mathrm{H}, J=2.4,11.5 \mathrm{~Hz}), 4.48(\mathrm{~m}, 2 \mathrm{H}), 7.38-7.71(\mathrm{~m}, 10 \mathrm{H})$, $9.88(\mathrm{~d}, 1 \mathrm{H}, J=2.4 \mathrm{~Hz})$.

4-O-Acetyl-2,3-O-isopropylidene-D-erythrose (1d). ${ }^{15}$ Yield: $89 \%$, colorless viscous oil; $[\alpha]_{\mathrm{D}}^{25}+31.8^{\mathrm{o}}\left(\mathrm{c} 1.0, \mathrm{CHCl}_{3}\right) ;{ }^{1} \mathrm{H} \mathrm{NMR}$ $\left(\mathrm{CDCl}_{3}\right) \delta 1.41(\mathrm{~s}, 3 \mathrm{H}), 1.59(\mathrm{~s}, 3 \mathrm{H}), 2.07(\mathrm{~s}, 3 \mathrm{H}), 4.02(\mathrm{~m}$, $1 \mathrm{H}), 4.40(\mathrm{dd}, 1 \mathrm{H}, J=2.3,12.0 \mathrm{~Hz}), 4.46(\mathrm{~m}, 2 \mathrm{H}), 4.59(\mathrm{~m}$, $1 \mathrm{H}), 9.71(\mathrm{~d}, 1 \mathrm{H}, J=2.3)$.

Acknowledgments. We thank the Minisry of Education, Science and Technology and the Korea Research Institute of Chemical Technology (KRICT) for the financial support.

\section{References}

1. (a) Wu, J. Z.; Gao, J.; Ren, G. B.; Zhen, Z. B.; Zhang, Y.; Wu, Y. Tetrahedron 2009, 65, 289-299. (b) Tachihara, T.; Kitahara, T. Tetrahedron 2003, 59, 1773-1780. (c) Kadota, K.; ElAzab, A. S.; Taniguchi, T.; Ogasawara, K. Synthesis 2000, 10, 1372-1374. (d) Sugahara, T.; Ogasawara, K. Synlett. 1999, 4, 419-420.

2. (a) Pawłowska, J.; Krawczyk, K. K.; Wojtasiewicz, K.; Maurin, J. K.; Czarnocki, Z. Monatsh Chem. 2009, 140, 83-86. (b) Oh, J. E.; Lee, K. H. Bioorg. Med. Chem. 1999, 7, 2985-2990. (c) Dickman, M.; Jones, J. B. Bioorg. Med. Chem. 2000, 8, 1957-1968.

3. (a) Joshi, B. V.; Moon, H. R.; Fettinger, J. C.; Marquez, V. E.; Jacobson, K. A. J. Org. Chem. 2005, 70, 439-447. (b) Gallos, J. K.; Stathakis, C. I.; Kotoulas, S. S.; Koumbis, A. E. J. Org. Chem. 2005, 70, 6884-6890.
4. (a) Moral, J. A.; Moon, S. J.; Torres, S. R.; Minehan, T. G. Org. Lett. 2009, 11, 3734-3737. (b) Bi, J.; Aggarwal, V. K. Chem. Commun. 2008, 120-122. (c) Kimura, M.; Ezoe, A.; Mori, M.; Iwata, K.; Tamaru, Y. J. Am. Chem. Soc. 2006, 128, 8559-8568. (d) Brabandt, W. V.; Vanwalleghem, M.; D'hooghe, M.; Kimpe, N. D. J. Org. Chem. 2006, 71, 7083-7086. (e) Liesener, F. P.; Jannsen, U.; Kalesse, M. Synthesis 2006, 15, 2590-2602. (f) Curti, C.; Zanardi, F.; Battistini, L.; Sartori, A.; Rassu, G.; Auzzas, L. Roggio, A.; Pinna, L.; Casiraghi. G. J. Org. Chem. 2006, 71, 225-230.

5. (a) Smith, A. B., III; Lin, Q.; Doughty, V. A.; Zhuang, L.; McBriar, M. D.; Kerns, J. K.; Boldi, A. M.; Murase, N.; Moser, W. H.; Brook, C. S.; Bennett, C. S.; Nakayama, K.; Sobukawa, M.; Trout, R. E. L. Tetrahedron 2009, 65, 6470-6488. (b) Guisot, N. A.; Lahoucine, B. O.; Canet, I.; Sinibaldi, M. E. Tetrahedron Lett. 2007, 48, 85118513. (c) Zarbin, P. H. G.; Oliveira, A. R. M.; Simonelli, F.; Villar, J. A. F. P.; Delay, O., Jr. Tetrahedron Lett. 2004, 45, 7399-7400.

6. (a) Jeong, B. S.; Choi, N. S.; Ahn, S. K.; Bae, H.; Kim, H. S.; Kim, D. Bioorganic \& Medicinal Chemistry Letters 2005, 15, 35803583. (b) Bennett, R. B., III; Choi, J. R.; Montgomery, W. D.; Cha, J. K. J. Am. Chem. Soc. 1989, 111, 2580-2582.

7. Mondiere, A.; Peng, R.; Remuson, R.; Aitken, D. J. Tetrahedron 2008, 64, 1088-1093.

8. Srihari, P.; Kumaraswamy, B.; Yadav, J. S. Tetrahedron 2009, 65, 6304-6309.

9. Li, Y. L.; Drew, M. G. B.; Welchman, E. V.; Shirvastava, R. K.; Jiang, S.; Valentine, R.; Singh, G. Tetrahedron 2004, 60, 65236531

10. (a) Evans, D. A.; Glorius, F.; Burch, J. D. Org. Lett. 2005, 7, 33313333. (b) Munier, P.; Krusinski, A.; Picq, D.; Anker, D. Tetrahedron 1995, 51, 1229-1244.

11. (a) Graham, T. J. S.; Gray, E. E.; Burgess, J. M.; Goess, B. C. J. Org. Chem. 2010, 75, 226-228. (b) Chen, W.; Liebeskind, L. S. J. Am. Chem. Soc. 2009, 131, 12546-12547. (c) Sibley, R.; Mokdad, H. H.; Schoenleber, R.; Musza, L.; Stirtan, W.; Marrero, D.; Carley, W.; Xiao, H.; Dumas, J. Bioorg. Med. Chem. Lett. 2003, 13, 19191922.

12. Munier, P.; Krusinski, A.; Picq, D.; Anker, D. Tetrahedron 1995, $51,1229-1244$

13. Evans, D. A.; Cee, V. J.; Siska, S. J. J. Am. Chem. Soc. 2006, 128, 9433-9441.

14. Storzl, T.; Bernet, B.; Vasella, A. Helv. Chim. Acta 1999, 82, 23802412.

15. Kenji, O.; Hideyuki, S. Noguchi Kenkyusho Jiho 1989, 32, 39-45. 